\title{
Editorial:
}

\section{Zika virus: raising awareness to prevent a pandemic}

ALAM MK $K^{1}$, ARSHAD AI

Keywords: Zika virus; Pandemic; ZIKV

Bangladesh Journal of Medical Science Vol. 17 No. 02 April'18. Page :327-328 DOI: http://dx.doi.org/10.3329/bjms.v17i3.36984

\section{Abstract:}

Zika virus is a flavivirus which is transmitted through Aedes female mosquito species or sexually. "Mild dengue" like symptoms are noticed which usually subside shortly and uneventfully. In 1969 ZIKV was isolated in West Malaysia for the first time. Since then no case has been reported in Malaysia. WHO has declared a global threat of an epidemic spread after the outbreak in Brazil. Rain forest climate regions are at a constant state of emergency as pools of water are abundantly formed as a result of rain showers throughout the year which provides a habitat for mosquitos. We try to raise awareness and alert health governing bodies and institutions to take necessary steps to prevent and eradicate the potential pandemic threat. Counselling of females who are planning to conceive is required to minimize the threat to newborn babies. Travellers visiting the equatorial regions should be screened and counselled to detect and prevent viral infection transfer and to avoid complications on a larger scale.

Zika virus (ZIKV), a form of flavivirus, is commonly thought to be transmitted through Aedes female mosquito species. Sexual transmission has also been documented. Symptoms of the viral infection are similar to those of dengue infection. Common symptoms are maculopapular rashes, arthralgia and abnormal lymphocytic activity. Symptomatic treatment and rest usually leads to a full recovery. However recently, congenital microcephaly has been ambiguously linked to infections during pregnancy.
During 1969, ZIKV was isolated in West-Malaysia ${ }^{1}$. Since then, no case in Malaysia has been documented as being affected directly or congenitally by ZIKV. In mid-2015, an epidemic outbreak originating from Brazil has been documented ${ }^{2}$. Coincidentally, outbreaks in Cambodia, Indonesia, French Polynesia, Malaysia and Philippines have also been reported ${ }^{3}$. Equatorial regions are at a constant risk of widespread infection attributing mainly to the rain forest climate. Malaysia has rain forest climate throughout the year. Increased risk of human-mosquito-human has been previously documented.

As it is a cultural melting pot of Asian continent and one of the favourite travelling destinations, peaks of tourism are noted in mid-year. World Health Organization has declared a state of emergency to control this epidemic from spreading across the globe. We request global health organizations such as UNICEF, WHO, etc. to join hands with Governments, Social Media, local NGOs, advertising agencies, and Health professionals to battle against the upcoming potential viral Pandemic. Serological testing and Polymerase Chain Reaction methods have been used for diagnosis of infection in early stages ${ }^{4}$.

Affected cases should be reported and studied to assess the risks of further spread. Females who plan to conceive or are pregnant should be closely monitored and counselled to avoid congenital transmission. Congenitally affected children should be assessed and provided necessary mental and health care facilities. The formulation of a vaccination is

1. Mohammad Khursheed Alam, Associate Professor, Orthodontic Department, College of Dentistry, Aljouf University. Sakaka, KSA.

2. Anas Imran Arshad, School of Dental Sciences, Universiti Sains Malaysia. Kota Bharu, Kelantan, Malaysia.

Correspondence to: Mohammad Khursheed Alam, Associate Professor, Orthodontic Department, College of Dentistry, Aljouf University. Sakaka, KSA. e-mail: dralam@gmail.com 
way down the lane and currently we should focus all efforts for preventing the spread of infection through minimizing the risk of exposure and controlling any further transmission of cases that have already been detected.

Within this context, the global model based on season as presented by Bogoch and colleagues ${ }^{2}$, predicts higher risks of local transmission of infection in equatorial regions throughout the year as well as travelers can worsen the global situation out of proportions. The potentiality of rapid spread of ZIKV requires an urgent notice and necessary preventive measures, viz., mosquito nets, repellent sprays, proper water sanitation, eradication of water pools, fumigation etc.

\section{References:}

1. Marchette, N., R. Garcia, and A. Rudnick, Isolation of Zika virus from Aedes aegypti mosquitoes in Malaysia. American Journal of Tropical Medicine and Hygiene, 1969. 18(3): p. 411-415.

2. Bogoch, II, et al., Anticipating the international spread of Zika virus from Brazil. Lancet, 2016. 387(10016): p. 335-336.
3. Wikan, N., et al., Immunological evidence of Zika virus transmission in Thailand. Asian Pacific Journal of Tropical Medicine, 2016. 9(2): p. 141-144.

4. Tappe, D., et al., Acute Zika virus infection after travel to Malaysian Borneo, September 2014. Emerging infectious diseases, 2015. 21(5): p. 911. 